\title{
Visualizing Green (GFP) and Red (DsRed) Fluorescent Proteins in Thin Sections with Laser Scanning Confocal and Transmission Electron Microscopy
}

\author{
P.A. Sims and J.D. Hardin,
}

Zoology Department, University of Wisconsin, 1117 West Johnson Street, Madison, WI. 53706

Fluorescent fusion proteins are widely used to visualize the localization of proteins in embryos and cultured cells. Our laboratory has fused several several $\mathrm{C}$. elegans $(\mathrm{Ce})$ epithelial junctional proteins to GFP and more recently, to DsRed. We sought to develop a technique to combine fluorescence detection with electron microscopy using the same thin section. Utilizing post-embedding fluorescence from 100nm (thin) sections and overlaying it with TEM images provides additional information not obtained by TEM imaging alone. Combined fluorescent-TEM images can be used to identify genotype, confirm patterns of TEM immunogold labeling; and serve as a visual reference for framing TEM images in a more familiar perspective. Visualizing fluorophores in plastic embedded thin sections is useful for low and high resolution localization, to find an area or orientation of interest by light microscopy prior to electron microscopy, and for correlative visualization of the same structures by both laser scanning confocal microscopy (LSCM) and TEM. We are also investigating fixation conditions that optimize preservation of ultrastructure with expression of fluorophores, as well as the incorporation of additional fluorescent probes to utilize the perdurance of fluorescent signal in thin sections.

Correlative imunofluorescence has been demonstrated using thin cryosections [1,2], and GFP has been visualized in $1 \mu \mathrm{m}$ sections [3]. The detection of fluorescent markers such as GFP or DsRed in thin sections has not been previously reported. The use of plastic embedded material has advantages over cryosections regarding ease of sectioning without specialized equipment and the ability to generate higher contrast images [3]. We demonstrate post-embedding fluorescence of GFP and DsRed in thin sections for correlative light and electron microscopy. High pressure freezing (HPF) $\mathrm{Ce}$ adults followed by embedding preserves fine structure and immuno-reactivity and freeze substitution in solvent with $1-5 \%$ water has been reported to improve visibility of membranes $[4,5]$. We combined HPF, freeze substitution, and low temperature embedding to observe AJM-1::GFP in thin sections and then immunogold labeled the same sections for TEM. AJM-1 localizes to the apical junction in epithelia and can be visualized in the hypodermis and intestine. Viewing fluorescent expression in thin sections by LSCM permits higher $\mathrm{z}$ resolution imaging than would otherwise be possible because thin sections are $100 \mathrm{~nm}$ thick, while LSCM z resolution is $500 \mathrm{~nm}$ [6]. Additional benefits of thin sections include sharper images and decreased background fluorescence when imaging with LSCM [1].

We used LSCM to image GFP expression and used a backscattered light (BSL) image to visualize the surface of thin sections on a coated finder grid. BSL is particularly useful to locate samples in sections and to remain focused on the surface of the grid while checking for fluorescence. The BSL image is simultaneously obtained and aligned with the fluorescent image. The merged fluorescent and BSL image is useful for aligning LSCM images with TEM images. TEM of these samples allowed us to correlate immunogold labeling with GFP expression from the same thin section (see figure 1). 
A monomeric variant of red fluorescent protein from coral, mDsRed has been described as showing faster maturation and reduced tetramerization than standard DsRed [7]. We constructed a strain expressing DLG-1::mDsRed, which co-localizes with AJM-1 to apical junctions in Ce epithelia [8]. Using chemical fixation and low temperature embedding in methacrylate resin (LR Gold), we were able to image DLG-1::mDsRed in post embedded thin sections by LSCM (see figure 2). We are currently working to immunogold label DsRed in thin sections.

References

[1] Robinson, JM, et. al., J. Histo Cyto. 49(7) (2001) 803-808.

[2] Takizawa T. and Robinson J. J. Histo Cyto. 51(6) (2003) 707-714.

[3] Luby-Phelps et. al., J. Histo Cyto. 51(3) (2003) 271-274.

[4] Rostaing,Phillippe et. al., J. Histo Cyto. 52(1) (2004) 1-12.

[5] Walther, P., Ziegler A., J. of Microscopy 208, Pt1. Oct 2002.

[6] Inoue S (1995). In Pawley, JB. ed. Handbook of Biological Confocal Microscopy.

[7] Campbell, R. E., (PNAS) vol. 99(12) (2002) 7877-7882.

[8] Koppen et. al. Nature Cell Biol. 3 (2001) 983-991.
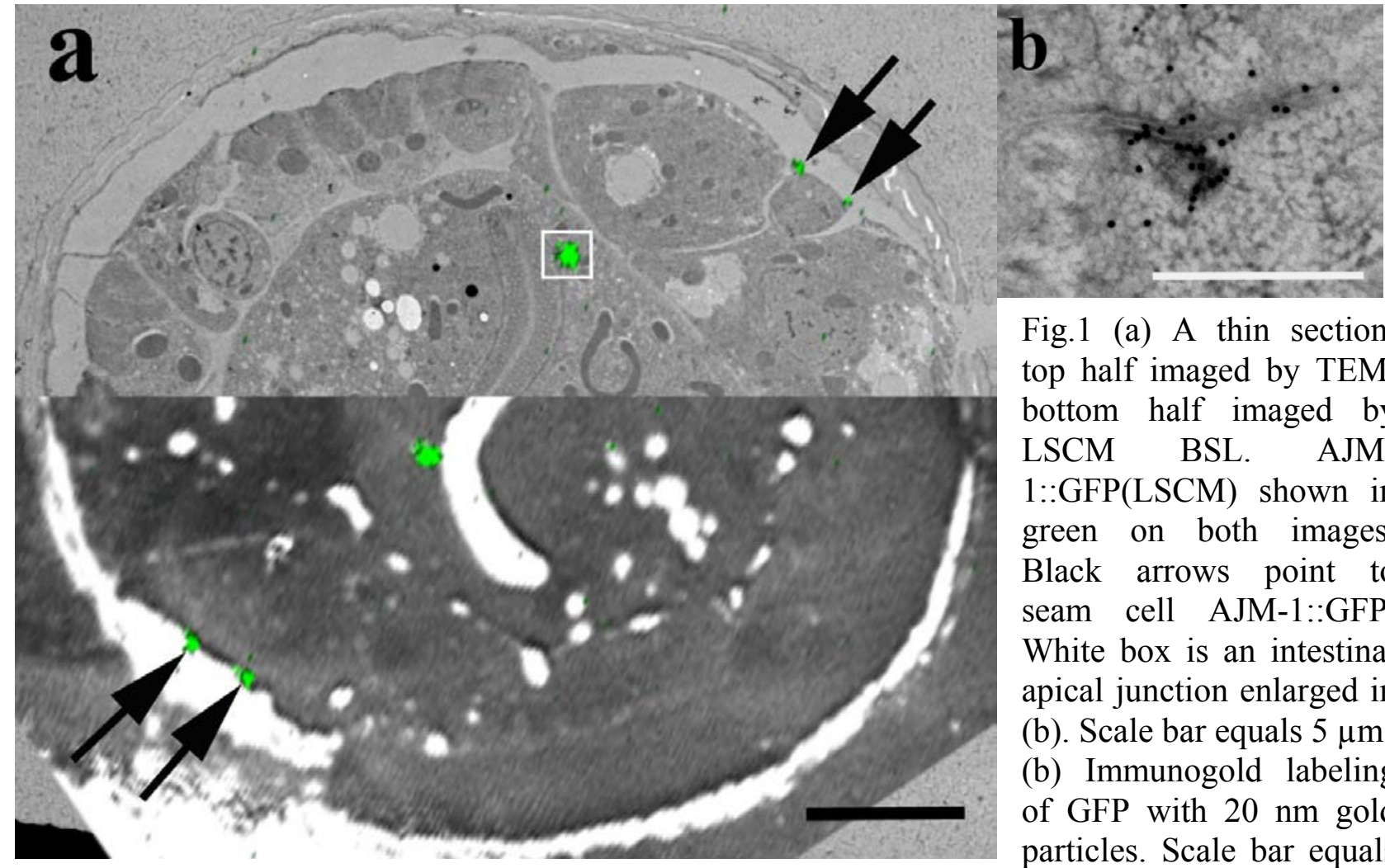

Fig.1 (a) A thin section, top half imaged by TEM, bottom half imaged by LSCM BSL. AJM$1:: \mathrm{GFP}(\mathrm{LSCM})$ shown in green on both images. Black arrows point to seam cell AJM-1::GFP. White box is an intestinal apical junction enlarged in (b). Scale bar equals $5 \mu \mathrm{m}$. (b) Immunogold labeling of GFP with $20 \mathrm{~nm}$ gold particles. Scale bar equals

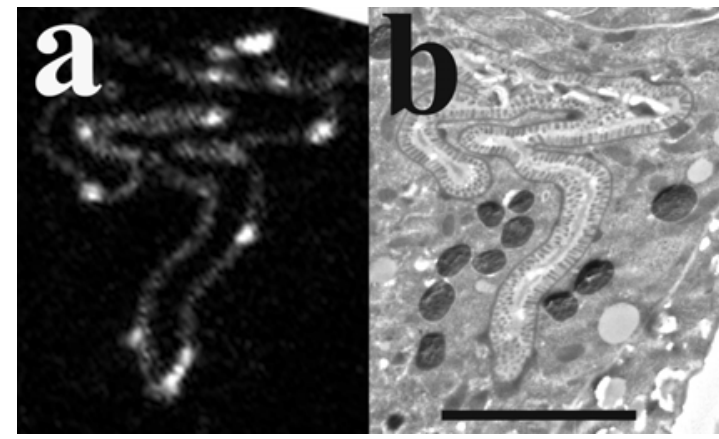
$500 \mathrm{~nm}$.

Fig. 2 (a) Intestinal DLG-1::mDsRed visualized in a thin section by LSCM. (b) A TEM image from the same area showing the intestinal expression of DLG$1:: \mathrm{mDsRed}$. Scale bar equals $5 \mu \mathrm{m}$ for both images. 